\title{
MODELACIÓN DISCRETA DE LA ESTRUCTURA DE TASAS DE INTERÉS NOMINALES, EL CASO DE COLOMBIA 2004 - 2013'
}

\author{
Francisco Javier Hernández Caballero²
}

Universidad de la Costa, CUC, Colombia - Artículo Tipo 1. Investigación Científica y Tecnológica - Recibido: 2 de Diciembre 2014 Aceptado: 12 de Junio 2015

\section{RESUMEN}

El estudio de las estructuras de tasas, desde una perspectiva teórica y empírica, siempre ha sido abordado con modelos en tiempos continuos. Esto puede ser explicado porque las primeras investigaciones al respecto, se dieron en mercados de capitales desarrollados. La funcionalidad de estas estructuras de tasas está dada por las expectativas económicas y financieras que refleja su dinámica; que se puede constatar con la interacción con variables como la inflación o la actividad económica de un país. Estas últimas, sin embargo se presentan con una dinámica discreta. En este trabajo se estimó para Colombia una estructura de tasas nominales en tiempo discreto, cuyo objetivo principal es encontrar bajo este contexto, la interacción teórica de las estructuras de tasas sobre ciertas expectativas económicas y financieras. Metodológicamente, la modelación se abordó con los típicos modelos afines que ofrece la literatura al respecto, en la versión dinámica y discreta propuesta por Alfaro(2011). Los resultados de la estimación permiten concluir que la modelación discreta con cotizaciones de tasas nominales de Títulos de Tesorería de Clase B, ajustan bastante bien y son un buen referente estadístico para contrastar las expectativas de inflación.

\section{Palabras Clave:}

Curvas de rendimiento, Modelos de ajustes, Expectativas de Inflación y Títulos de Tesorería -TES-.

JEL: G31, G32, P24

Si va a referenciar este artículo

Hernández, F. (2015). Modelación discreta de la estructura de tasas de interés nominales, el caso de Colombia 2004 - 2013, Económicas CUC, 36 (1), 95-111

\footnotetext{
${ }^{1}$ Artículo de investigación, dentro de la línea de Economía Financiera, producto de la investigación sobre Teoría, Aplicación, Modelado y Funcionalidad de Las Estructuras de Tasas de Interés Nominales, que el autor viene desarrollando a partir de su trabajo de investigación con el cual se tituló como Magister en Economía Financiera en la Universidad de Santiago de Chile.

${ }^{2}$ Contador Público y Economista, Especialista en Finanzas y Magister en Economía Financiera. Docente investigador Universidad Simón Bolívar y Corporación Universitaria Americana. Barranquilla Colombia. fhernandez@ unisimonbolivar.edu.co
} 


\section{INTRODUCCIÓN}

Teórica y empíricamente, la construcción de modelos para ajustes de curvas de rendimiento o Estructuras de Tasas de Interés (ETTI), han girado en torno a dos expectativas. Primero en torno a lo económico, puesto que la dinámica expresada en su construcción implícitamente debe reflejar las expectativas que los agentes del mercado tienen sobre los títulos soberanos (Rojas, 2007), sobre los cuales recae o es instrumentada la política económica, como la monetaria y fiscal, del Estado que emite dichos títulos.

Segundo, en lo financiero, su funcionalidad se acentúa en la valoración de activos, evaluación de riesgos, diseño de estrategias de cobertura (Romero \& Robles, 2003); referenciar el costo del dinero en función del tiempo (Cámaro, Henao \& Mendez, 2005) y entre otros, ser referente para establecer rentabilidades en cualquier sector de los mercados de deuda: empresarial, bancaria, internacional 0 municipal y valoración de activos reales. (Marin \& Rubio, 2011)

Atendiendo a las funcionalidades anteriores, con base en el trabajo de Alfaro (2011), en el presente documento se construyó la ETTI en forma discreta, tomando las cotizaciones de los títulos de tesorería clase B, emitidos por el Estado colombiano. A diferencia de trabajos similares como Melo \& Castro (2010), quienes ajustan la curva en forma continua y la relacionan con variables dadas por el mercado como la inflación y la brecha del producto, en éste se contrasta con variables en expectativas.

En este trabajo, además de esta introducción, en la primera parte se enmarcan los referentes teóricos para la derivación de las curvas de rendimientos puntualizando los principales enfoques. En la segunda se describe la pertinencia de los datos utilizados, en la tercera se hace la respectiva modelación de los datos, en forma econométrica y en la cuarta parte se expresan los principales resultados y conclusiones.

\section{REFERENTE TEÓRICO}

A través del tiempo, los economistas financieros percibieron que los rendimientos que exigían los títulos de renta fija, variaban en función del plazo al vencimiento (Dumrauf, 2015). Con esto, la ETTI o curvas de rendimiento son entendidas como la relación que existe entre el tiempo que falta hasta el vencimiento de los títulos de renta fija, y los rendimientos de estos mismos títulos. De hecho, los mismos economistas financieros interpretaron la funcionalidad inmersa en esta relación. Por lo tanto, la forma de modelar esta relación ha sido de gran interés para el quehacer de las finanzas.

Las investigaciones, teóricas y empíricas sobre la ETTI, en principio se centraron en encontrar los mejores ajustes en ausencia de arbitraje, y cuando al respecto hubo el suficiente consenso, los estudios se extendieron a la interacción entre la estructura de tasas y variables macroeconómicas y financieras.

Se han abordado desde escenarios paramétricos y no paramétricos, destacándose los de Nelson \& Siegel (1987); Svensson (1994), Polinomios Trigonométricos y Locales, Splines ${ }^{3}$ Suavizados, Componentes Principales, Análisis Factorial, Redes Neuronales, entre otros.

Santana (2008), ofrece una completa revisión metodológica con la cual aproxima la curva de rendimiento a los mercados de Colombia y Estados Unidos. A su vez, Ro-

${ }^{3}$ Curvas diferenciables definidas en porciones mediante polinomios. 
mero \& Robles (2003), hacen una revisión teórica y empírica, tanto de los métodos como de los modelos (enfoques macroeconómicos y financieros) con que se han abordado los estudios.

Normalmente, las estimaciones de las Estructuras de Tasas de Interés han sido ajustadas con modelos de factores ${ }^{4}$, o afines, de los cuales el de Vasicek (1977) y Cox, Ingersoll, \& Ross, (1985), son acotados a no presentar oportunidades de arbitraje y son generados en procesos estocásticos. (Alfaro, 2009)

Cox et al., (1985), derivan un modelo de un solo factor para describir la estructura temporal, el cual lo desarrollan en un contexto de modelación de valoración de activos en equilibrio general inter temporal.

Vasicek (1977), argumenta que bajo la ausencia de arbitraje, (entre otros supuestos) la tasa de rendimiento esperada para cualquier bono mayor que la tasa spot (Tipo de cambio que se aplica en las compras inmediatas de divisas), es proporcional a su desviación estándar.

El paradigma de mayor referencia lo constituye el modelo de ajuste de Nelson \& Siegel (1987), quienes introducen un ajuste paramétrico, también en tiempo continuo, para determinar la curva de rendimientos. Sus argumentos, se basan en que las formas de estas curvas están asociadas a las soluciones de ecuaciones en diferencias y que la teoría de las expectativas de la estructura de las tasas de interés, provee una motivación heurística para investigar tal sentido de ajuste. El modelo establece que las tasas forwards (futuras) presentan la siguiente forma:

(1) $R(m)=\beta_{0}+\beta_{1} \frac{\left(1-e^{-m / t}\right)}{m / t}+\beta_{2}\left[\frac{\left(1-e^{-m / t}\right)}{m / t}-e^{-m / t}\right]$

Esta forma de extraer las curvas de rendimientos no es lineal, pero para efectos empíricos de contextualizar la modelación econométrica, se asume que el parámetro t se conoce. Así mismo, del modelo se desprende que estimaciones en las cuales se hace que la madurez sea larga, el rendimiento estaría dado por el factor $\boldsymbol{\beta}_{0} \mathrm{y}$ esto sería interpretado como la tasa larga. Estimaciones a corto plazo, $\boldsymbol{m}$ muy pequeño, la estructura se hace con los factores $\boldsymbol{\beta}_{0}+\boldsymbol{\beta}_{1}$ que implicaría la tasa corta.

La popularidad y acogimiento institucional en muchos países, del modelo de Nelson \& Siegel (1987), viene dada por lo parsimonioso o simple, y flexible que resulta para modelar cualquier forma de la curva de rendimiento.

Svensson (1994), presenta una extensión del modelo de Nelson \& Siegel (1987), agregando un cuarto factor $\left(\boldsymbol{\beta}_{3}\right)$ semejante al tercero $\left(\boldsymbol{\beta}_{2}\right)$, con lo cual la tasas forward vendrían dadas por el siguiente modelo:

$$
R(m)=\beta_{0}+\beta_{1} \frac{\left(1-e^{-m / t_{1}}\right)}{m / t_{1}}+\beta_{2}\left[\frac{\left(1-e^{-m / t_{1}}\right)}{m / t_{1}}-e^{-m / t_{1}}\right]+\beta_{3}\left[\frac{\left(1-e^{-m / t_{2}}\right)}{m / t_{2}}-e^{-m / t_{2}}\right]
$$

\footnotetext{
${ }^{4}$ Básicamente esto implica obtener parámetros asociados al tiempo como variable, el cual es reemplazado al vencimiento que se desee estimar.
} 
La presencia del cuarto factor ( $\beta 3$ ) contribuye a tener un ajuste más refinado, sobre todo en los tramos de largo plazo, pues provee un efecto adicional y semejante al segundo factor. En algunos escenarios, cuando la estructura de tasas es más compleja, el modelo de Svensson, se desempeña mejor que el de Nelson \& Siegel. (Santana, 2008)

Diebold \& Li (2006), presentan una re parametrización del modelo de Nelson \& Siegel (1987). Ofrecen una interpretación más moderna de los factores entendiéndolos como factores dinámicos latentes. Concluyen que lo que en la literatura se ha interpretado como estimaciones de largo, mediano y corto plazo, derivados de los límites estos mismos, también pueden ser interpretados en términos de nivel (level), pendiente (slope) y curvatura (curvature).

Otra caracterización importante que le dan Diebold \& Li (2006), al modelo de Nelson- Siegel (1987), es la caracterización dinámica, en términos de econometría de series de tiempo, por lo cual se le conoce como Nelson-Siegel Dinámico. Christensen, Diebold, \& Rudebusch (2010), encuentran que esta versión dinámica, en un contexto de no arbitraje pertenece a la clase de modelos afines o los típicos modelos de factores.

Teórica y empíricamente todos los modelos de ajustes de las curvas de rendimiento, se han basado en un escenario de tiempo continuo. Sin embrago, recientemente Alfaro (2011), propone una versión discreta del Nelson-Siegel Dinámico, y en este sentido, en paralelo con Christensen et al., (2010), demuestra que el modelo pertenece a la clase de modelos afines. Tras lo anterior, Alfaro (2011), apoyado con aproximaciones de Taylor deriva la versión discreta del mo- delo Nelson-Siegel dinámico de la siguiente manera ${ }^{5}$ :

$$
R(m)=\beta_{0 t}+\frac{\beta_{1 t}}{m}\left[\frac{1-\phi^{m}}{1-\phi}\right]+\frac{\beta_{2 t}}{m}\left[\left(\frac{1-\phi^{m}}{1}\right)-m \phi^{m-1}\right]
$$

Una de las ventajas teóricas y esperadas de esta versión, sobre todo por los propósitos del presente trabajo, es el poder trasladar el análisis de interacción con otras variables que no presentan un comportamiento continúo, a un escenario donde las variables estrían vis a vis ${ }^{6}$ en términos del comportamiento de frecuencias. Pueden analizarse series (económicas y/o financieras) con frecuencia mensual, o trimestral, según el caso, con una estructura de tasas derivadas en frecuencia mensual o trimestral, de nuevo, según el caso.

\section{Mercado de TES y datos estilizados}

Es natural construir la ETTI con bonos gubernamentales, que sólo se diferencien en el plazo de vencimiento. El uso de estos instrumentos es justificado porque i) no presentan riesgo de insolvencia, ii) tienen una alta liquidez y un gran volumen de negociación por lo que sus precios reflejan la información disponible en el mercado (Marin \& Rubio, 2011), y iii) tienen un grado semejante de fiscalidad. (Mascareñas, 2013)

Con base en la teoría de derivación de curvas de rendimiento con títulos soberanos-

\footnotetext{
5 Para mayor detalle ver los siguientes documentos referenciados: Alfaro, Becerra, \& Sagner, (2011) y Alfaro (2009)

${ }^{6}$ Expresión española procedente del francés vis-à-vis, que significa 'cara a cara'.
} 
deuda pública - por cumplir con el rol de activos libre de riesgo, de crédito - se trabajó con datos de las cotizaciones de tasa de los Títulos de Tesorería - TES de Clase B, emitidos por el gobierno central colombiano, enmarcados en la ley 51 de 1990.

Según datos del Ministerio de Hacienda, la dinámica de estas emisiones de TES ha mantenido un crecimiento constante, pasando de $38,95 \%$ de la deuda total en diciembre de 2001, a 71,27\% a julio de 2013. De igual manera, en la deuda interna pasó de $81,41 \%$ al $98,34 \%$ entre junio de 2001 y julio de 2013. En ambos casos, los TES tienen mayor participación en toda la dinámica.

De las diferentes formas de TES, las emitidas en pesos a tasa fija son las que siempre han tenido mayor representatividad, incluso también han tenido una dinámica creciente, pues, según información de deuda pública del Ministerio de Hacienda, han pasado de representar el $37,90 \%$ al $77,48 \%$ de junio de 2001 a julio de 2013.

En complemento a lo anterior, se trabajó con las cotizaciones de tasas de los TES de clase B, por, i) su importancia relativa tanto en la deuda total como interna, ii) los objetivos con los cuales fueron creados tienen mayor dinámica de emisión; es decir sus emisiones son más frecuentes respecto de los de clase A, que ya prácticamente no se emiten, iii) atendiendo a esas mismas características de creación, y por los plazos a los cuales se emiten, podría argüirse que también han sido creados para desarrollar el mercado de capitales en Colombia y dar señales de las expectativas de precios y tasas por plazos, lo que los hace ideales para la construcción de las curvas de rendimiento.
Para reforzar el porqué de la utilización de los TES de clase B, expresados en pesos y negociados a tasa cupón fija y no segregables, Reveiz \& Leon (2008), con el fin de contar con un Benchmark ${ }^{7}$ para el mercado de deuda pública interna colombiano, ofrecen un índice representativo, describiendo los principales fundamentos y principios metodológicos reconocidos a nivel internacional, para la construcción de tales índices y los ajustan al contexto colombiano. En sujeción a lo anterior, Reveiz \& Leon (2008), para la calibración del índice Benchmark, reportan la utilización exclusivamente de los TES de clase B, expresados en pesos, a tasa fija y negociados no en forma segregable.

\section{Modelación econométrica}

En este apartado se exponen dos escenarios, uno en donde se estima el modelo Nelson-Siegel Dinámico en la versión discreta propuesta por Alfaro (2011). En un segundo escenario se contrasta la interacción de la dinámica de la estructura de tasas con las expectativas de inflación y de actividad económica en Colombia.

Para la primera etapa, se trabajó con las tasas de cierre del último día hábil de cada mes. La tabla 1 muestra la participación de las diferentes tasas con las cuales se trabajó. Es pertinente anotar, que para los vencimientos más cortos, por ser plazos a cumplir muy próximos, por lo general presentan pocas transacciones y por ende pocas cotizaciones reales de tasas, por lo que algunos autores acuden a diferentes clases de títulos, tomando como tasas cortas las del mercado monetario.

\footnotetext{
7 En finanzas, referencia que se usa para hacer comparaciones.
} 
Tabla 1.

Participación de los Diferentes Vencimientos

\begin{tabular}{lccc}
\hline \multicolumn{1}{c}{ Plazos } & Frecuencia & Porcentaje & Acumulado \\
\hline 0 a 3 vencimientos & 61 & $3,40 \%$ & 3,41 \\
\hline Más de 3 y menos de 6 Meses & 74 & $4,10 \%$ & 7,55 \\
\hline Más de 6 y Menos de 12 Meses & 156 & $8,70 \%$ & 16,28 \\
\hline Más de 12 y Menos de 24 Meses & 290 & $16,20 \%$ & 32,51 \\
\hline Más de 24 y Menos de 36 Meses & 256 & $14,30 \%$ & 46,84 \\
\hline Más de 36 y Menos de 60 Meses & 314 & $17,60 \%$ & 64,41 \\
\hline Más de 60 y Menos de 96 Meses & 244 & $13,70 \%$ & 78,06 \\
\hline Más de 96 y Menos de 120 Meses & 137 & $7,70 \%$ & 85,73 \\
\hline Más de 120 Meses & 255 & $14,30 \%$ & $\mathbf{1 0 0 \%}$ \\
\hline Total & $\mathbf{1 . 7 8 7}$ & $\mathbf{1 0 0} \%$ & \\
\hline
\end{tabular}

Fuente: Elaboración propia con datos del Sistema Electrónico de Negociación (SEN) administrado por el Banco de la República

\section{Estimación del modelo Nelson-Siegel dinámico en forma discreta}

En el primer escenario el modelo de la ecuación 3 fue estimado en dos etapas. En la primera se estimó para cada periodo (mes), a través de mínimos cuadrados ordinarios mediante una grilla para el parámetro do en un rango entre 0,70 hasta 0,99 con intervalos de 0,01 . El propósito de esto es encontrar en ese rango el dp que, bajo la medida de ajuste global $\mathrm{R}^{2}$ en el promedio de toda la muestra, optimice este $\mathrm{R}^{2}$. De la misma manera se promedió el error estándar para toda la muestra. Los resultados, presentados en las Figuras 1 y 2 evidencian que para el $\mathrm{R}^{2}$ el do que lo optimiza en promedio a lo largo de la muestra es 0,95 y para el error estándar es cuando el do es igual a 0,93 . Con estos resultados, se trabajó la segunda etapa con un do intermedio de 0,94, el cual es idéntico al calibrado por Diebold \& Li, (2006) ${ }^{8}$. Esto implica que teniendo un do dado para la muestra, el modelo puede ser estimado por mínimos cuadrados ordinarios lineales.

\footnotetext{
8 Como quiera que se está trabajando con el modelo discreto de Alfaro (2011), con la respectiva transformación a tiempo continuo se estaría en armonía con Diebold \& Li (2006) que calibran $\lambda$ de 0,0609 .
}

Las Figuras 3, 4 y 5 muestran la dinámica de los factores de la curva de rendimiento. Diebold \& Li (2006), consideran como proxis empíricas de los factores, las siguientes combinaciones. El nivel como las tasas a largo plazo, es decir a 120 meses, asociado al tramo largo de la curva proponen la pendiente de la curva como la diferencia entre la tasa más corta y el nivel, siendo la tasa corta la estimación a 3 meses. Y definen la curvatura, asociada al tramo corto, como 2 veces la tasa a 24 meses, menos la tasa a 3 meses y menos la tasa a 120 meses.

Con la derivación de los factores de la curva de rendimiento, es pertinente evaluar qué tan bondadoso es el ajuste. En este sentido, se derivan tres curvas para tres periodos de la muestra, ubicando al principio, al medio y al final de toda la serie (Agosto 2004, Octubre 2008 y Junio 2012 respectivamente). Como vencimientos proyectamos los mismos para esos periodos y se comparan con las tasas reales para observar que tan bondadoso es el ajuste del modelo. Las Figuras 6,7 y 8 , evidencian que el modelo ajusta razonablemente bien la dinámica de las tasas futuras de vencimientos 


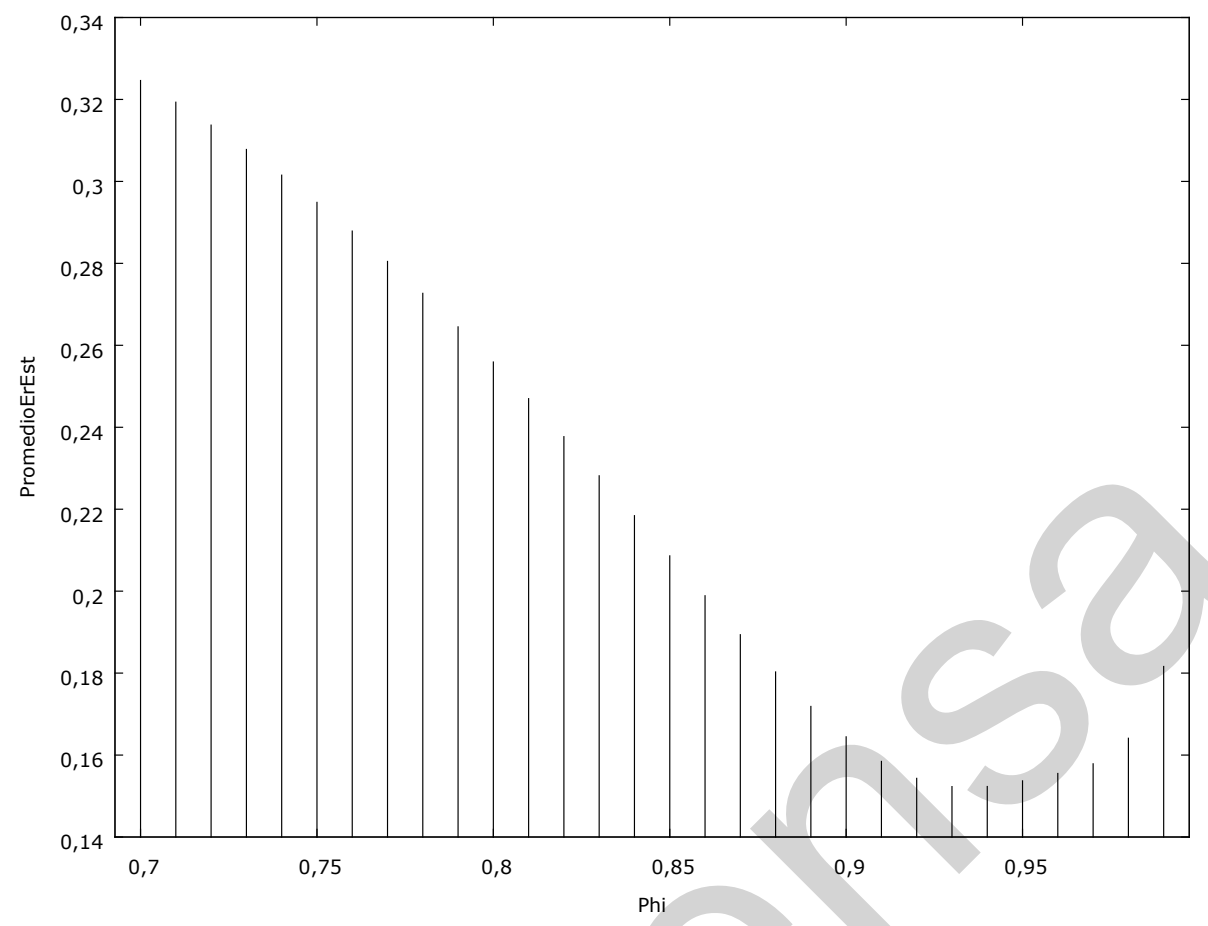

Figura 1. $\mathrm{R}^{2}$ Versus el Phi

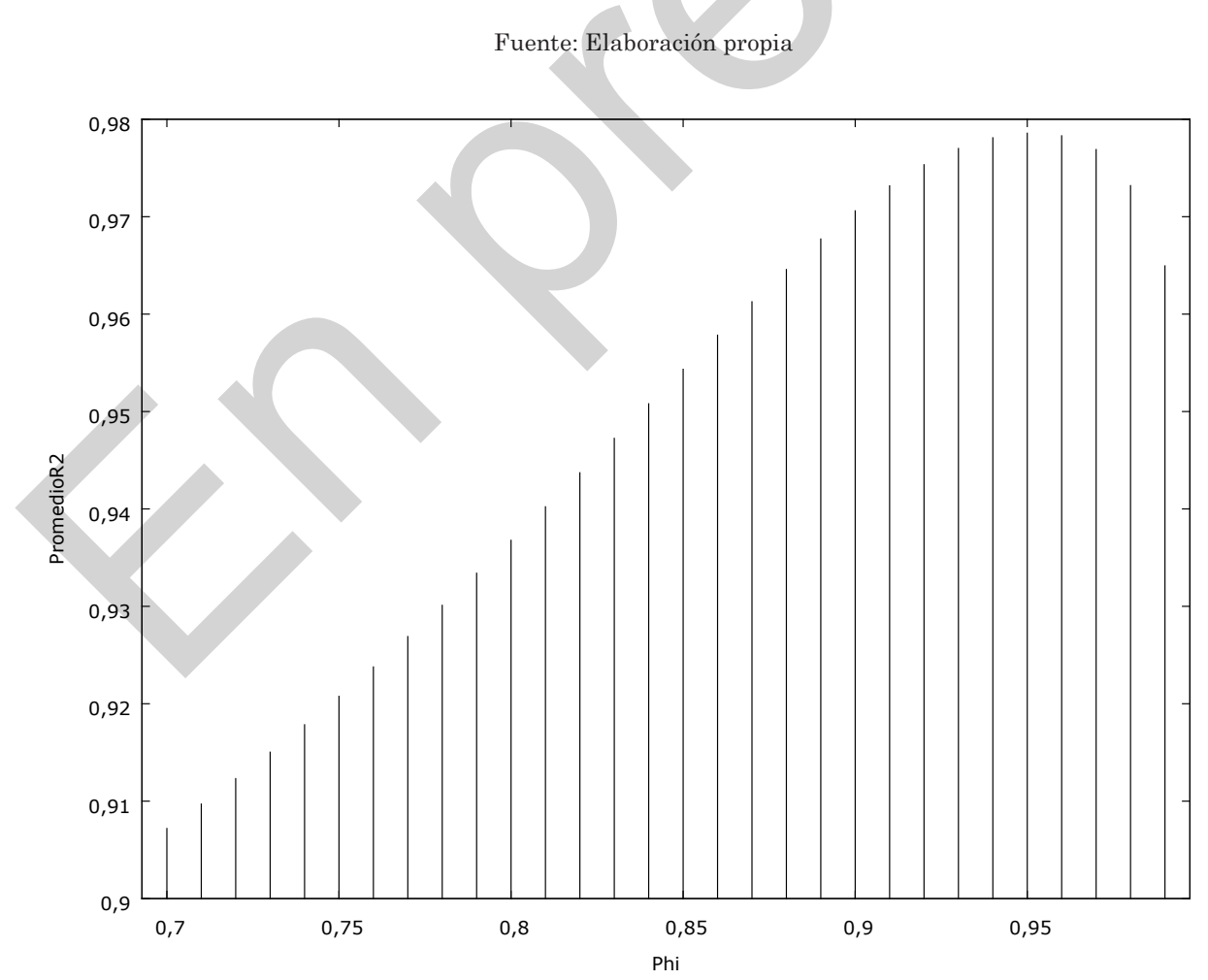

Figura 2. Error Estándar Versus el Phi

Fuente: Elaboración propia 


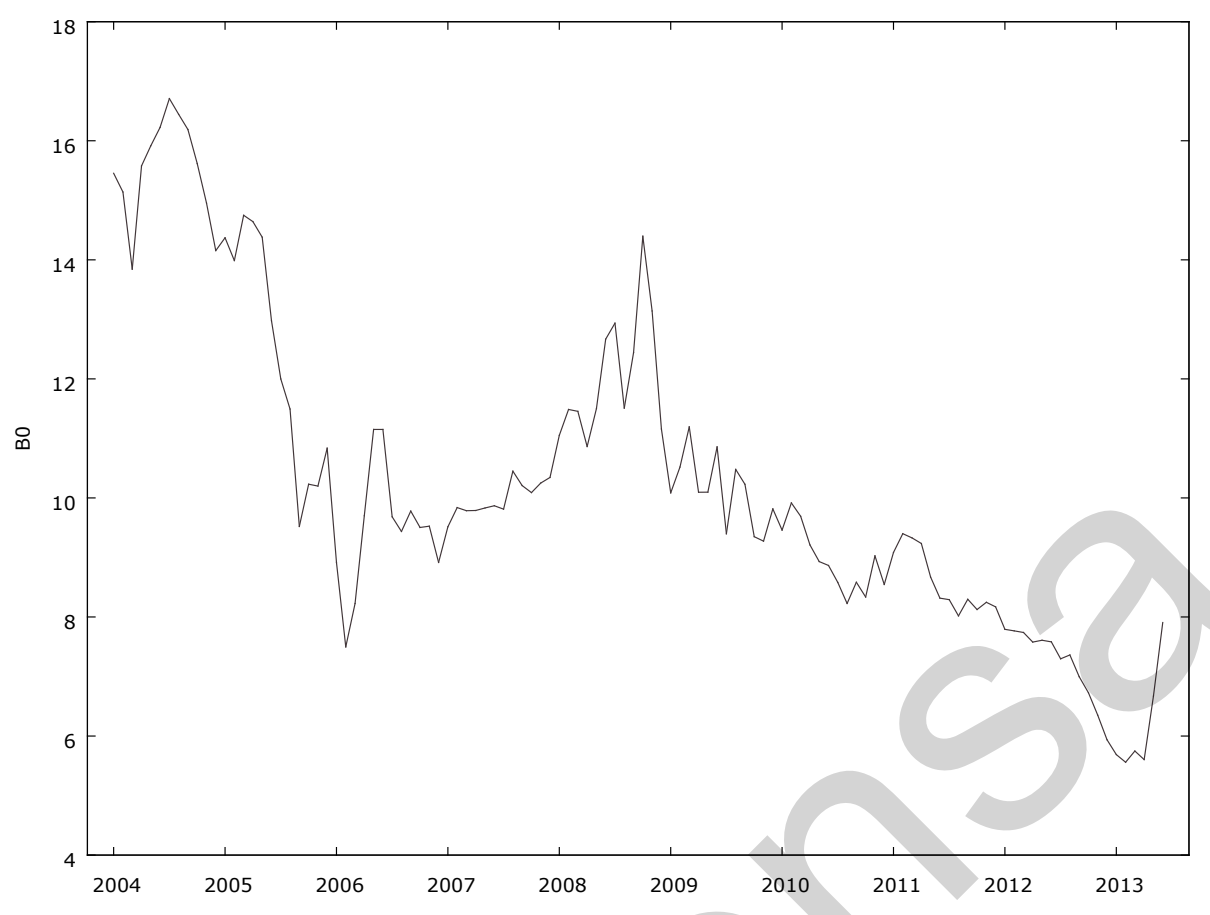

Figura 3. Nivel del Modelo

Fuente: Elaboración propia

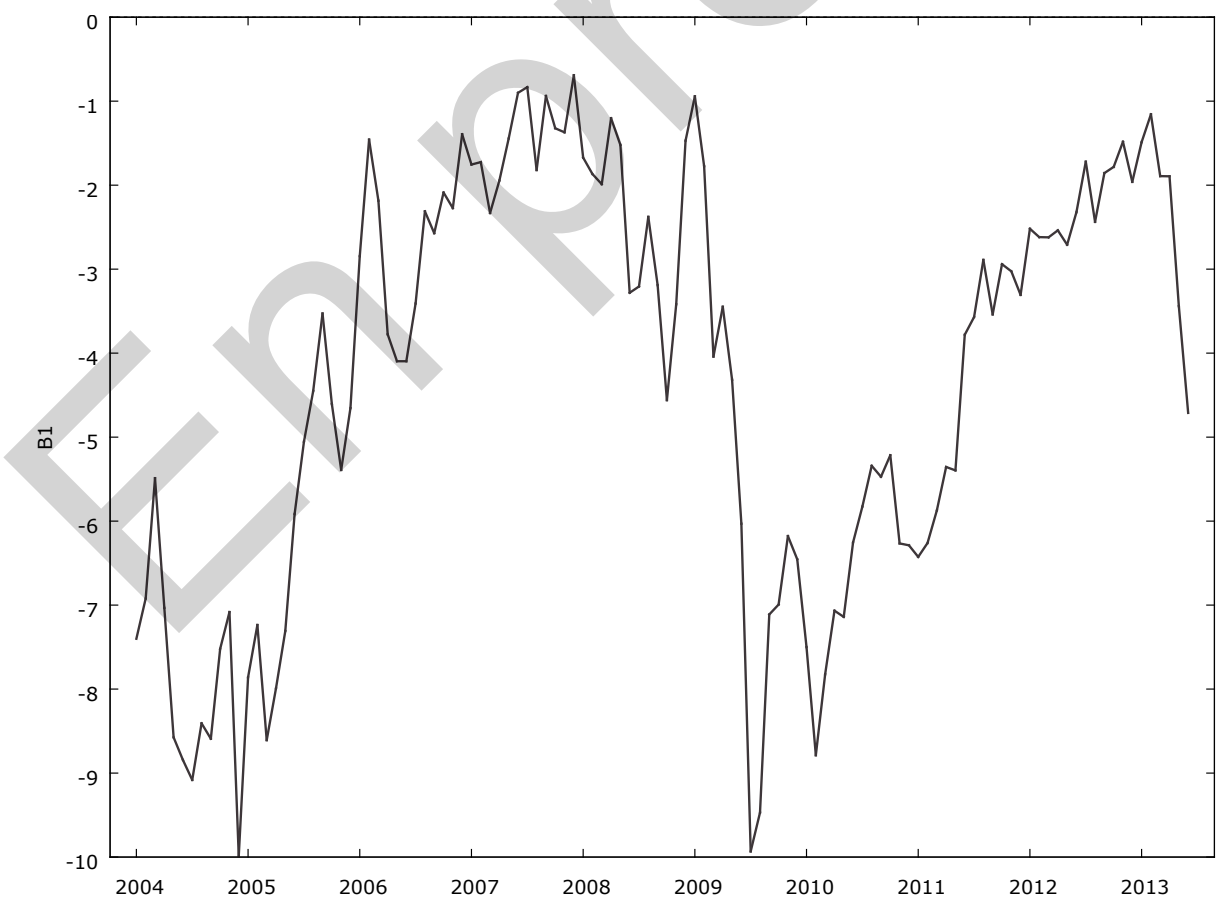

Figura 4. Pendiente del Modelo

Fuente: Elaboración propia 


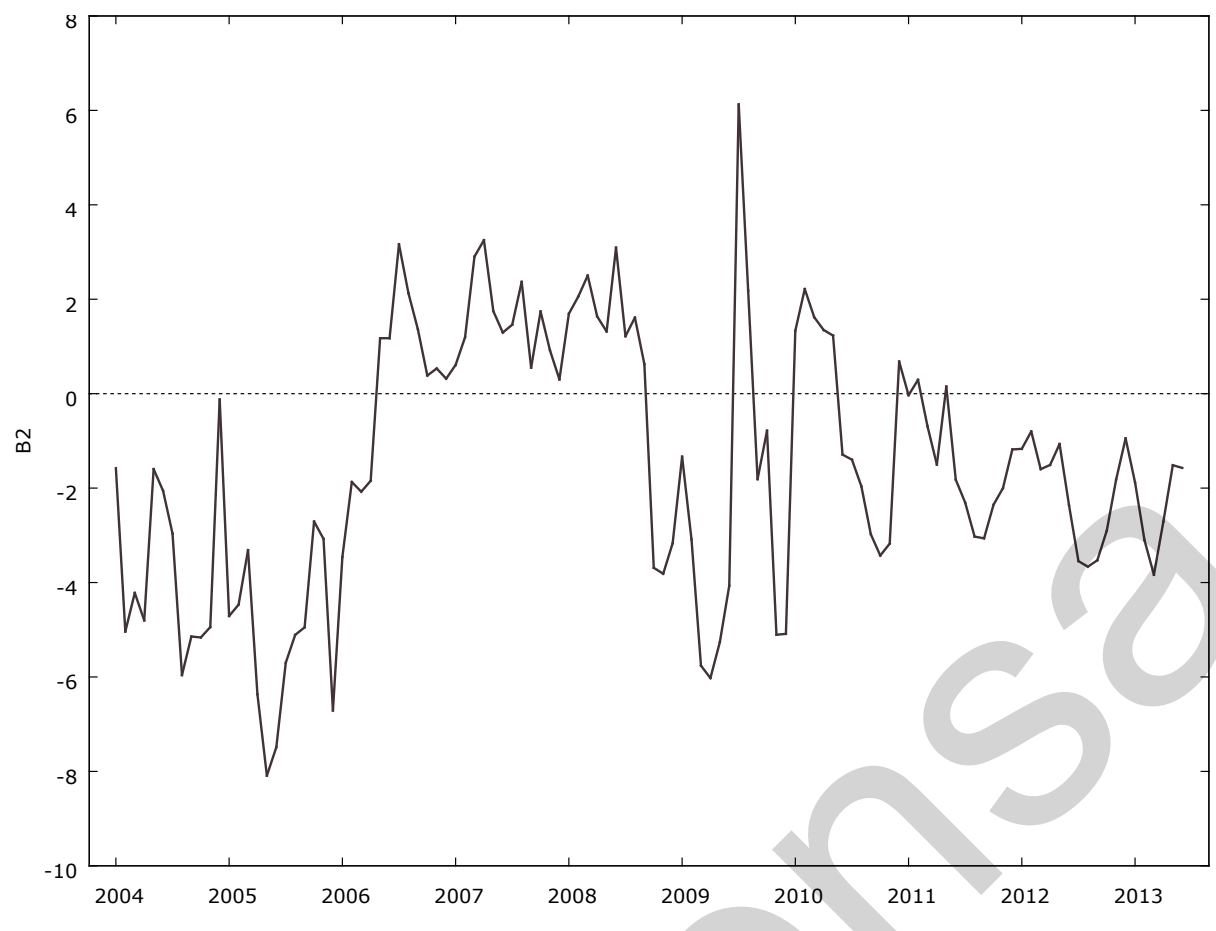

Figura 5. Curvatura del Modelo

Fuente: Elaboración propia

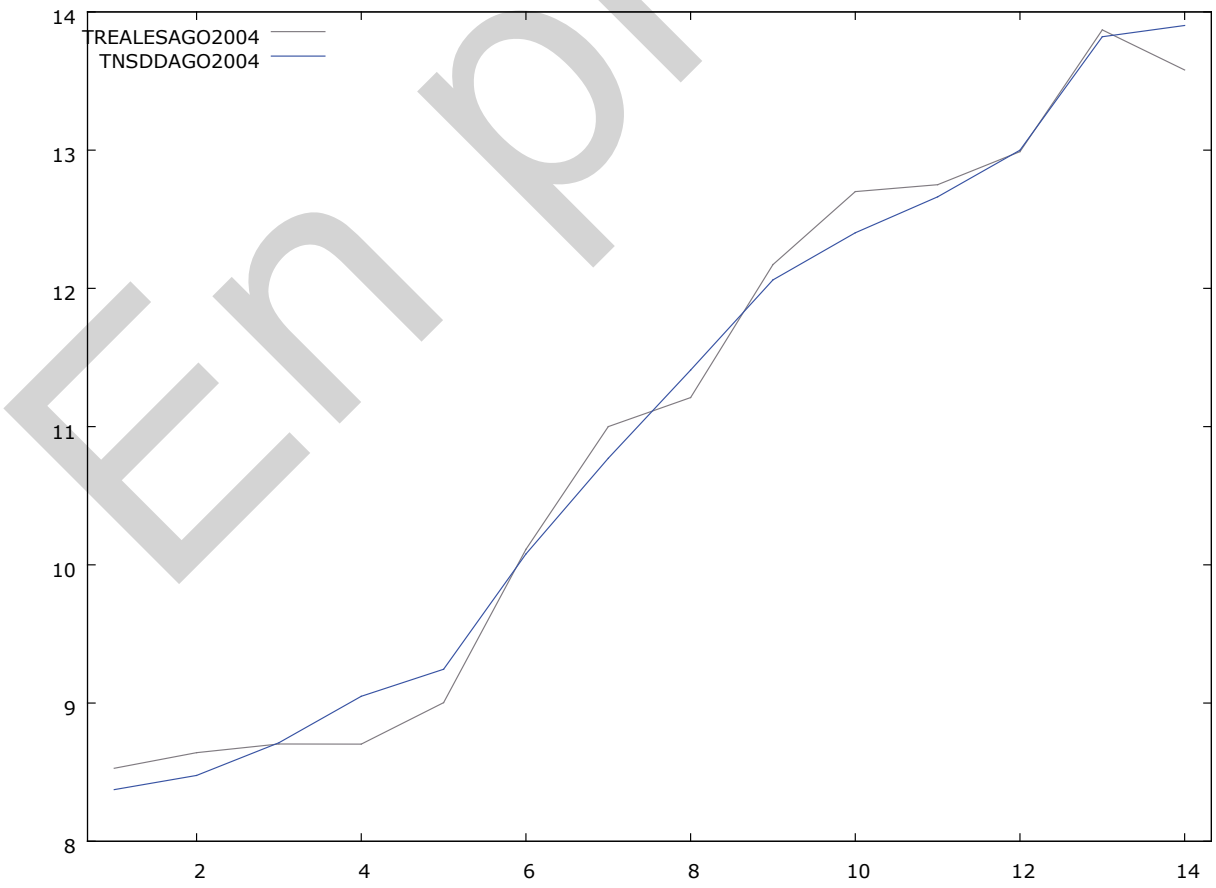

Figura 6. Curva de Rendimiento Modelada y Vencimientos Reales de Agosto de 2004 Fuente: Elaboración propia 


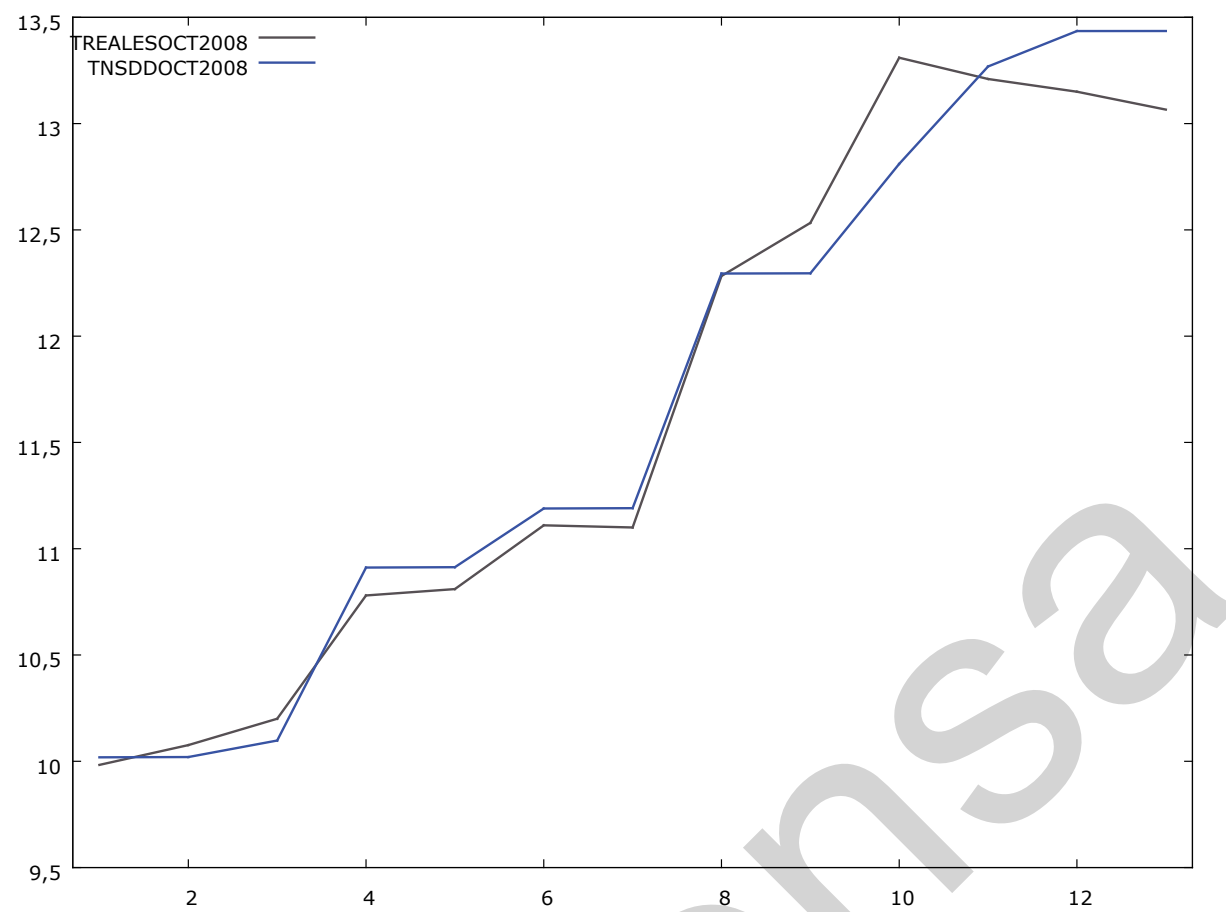

Figura 7. Curva de Rendimiento Modelada y Vencimientos Reales de Octubre de 2008 Fuente: Elaboración propia

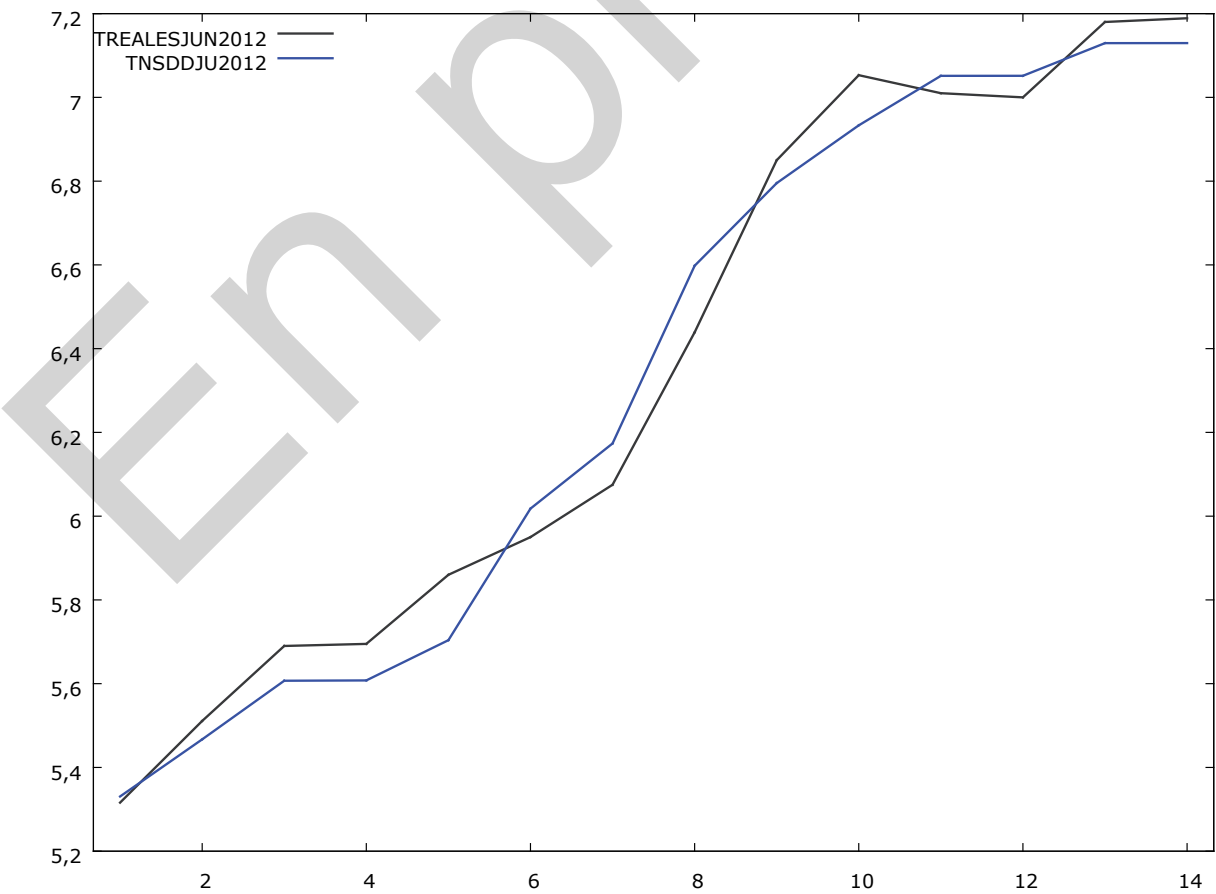

Figura 8. Curva de Rendimiento Modelada y Vencimientos Reales de Junio de 2012

Fuente: Elaboración propia 


\section{Estructura de tasas discretas y expectativas de inflación y actividad económica}

En esta sección, básicamente se analiza la interacción entre la dinámica de los factores de la estructura de tasas, obtenidas anteriormente, $y$ las expectativas de inflación y de actividad económica. Este análisis se aborda a través de estimaciones de tipoVAT(p).

Desde un contexto esencialmente macroeconómico - por las variables que interactúan con la estructura de tasas. Diebold, Rudebusch \& Aruoba (2006), estudian las relaciones entre el nivel con la inflación, y la pendiente con la brecha del producto. Rudebusch \& Wu (2004), lo hacen entre el nivel y las expectativas de inflación y la pendiente con la tasa de política monetaria. Desde esta perspectiva, para este estudio de caso, las figuras 9 y
10 muestran el comportamiento entre el nivel y las expectativas de inflación a diciembre de cada año y entre la pendiente y las expectativas del Índice Mensual de Actividad Económica (IMACO).

En el Tabla 2, se muestran los detalles de las series utilizadas entre enero de 2004 a junio de 2013. Para efectos de los análisis de equilibrio, apoyados en el test de Phillips-Perron solo la curvatura $\left(\boldsymbol{\beta}_{2}\right)$ no presenta raíz unitaria, mientras que las demás, con el mismo test, muestran un p-valor superior a 0,10 dando indicios de presencia de raíz unitaria (Ver Anexo No 1), las cuales al ser diferenciadas presentan un p-valor nulo, por lo que para efectos de predicción podrían ser tratadas $\left.I()_{1}\right)$. Sin embargo, atendiendo a que lo se busca es observar la estructura de relación, por las razones que se argumentan más adelante, las variables serán modelas en el VAR en su estado natural.

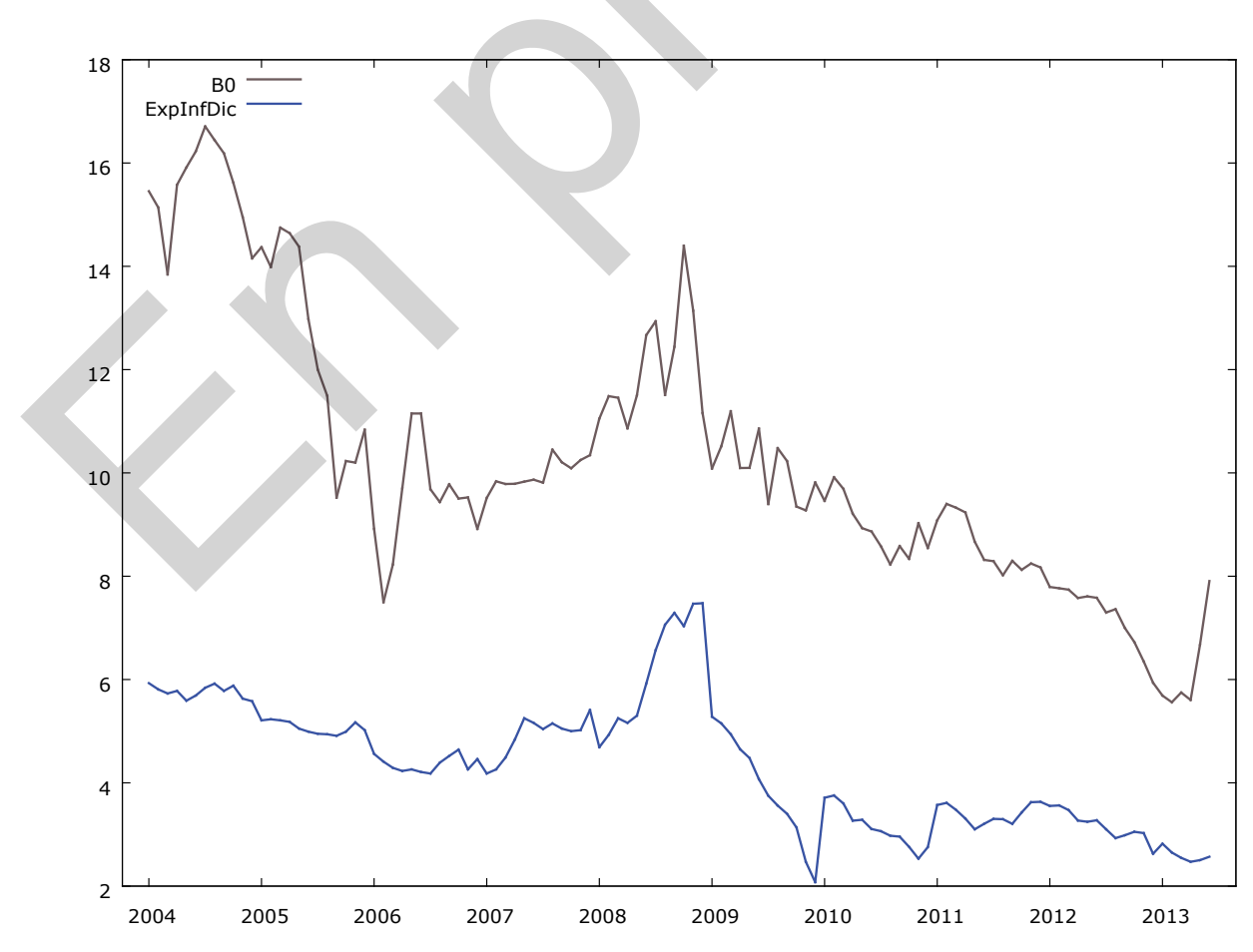

Figura 9. Nivel Versus Expectativas de Inflación a diciembre 


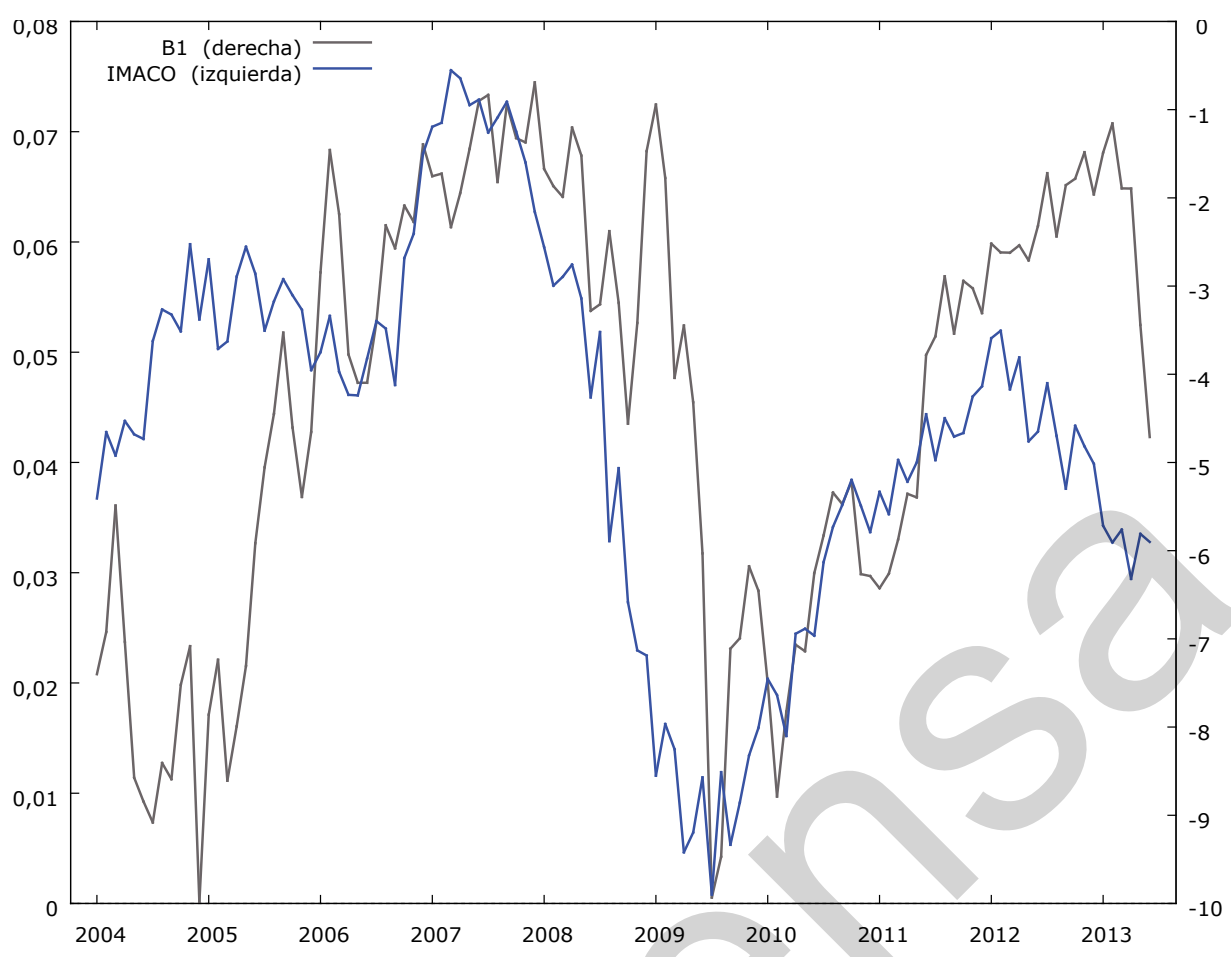

Figura 10. Pendiente Versus IMACO

Fuente: Elaboración propia

Tabla 2.

Series Utilizadas

\begin{tabular}{cll}
\hline Serie & \multicolumn{1}{c}{ Descripción } & \multicolumn{1}{c}{ Fuente } \\
\hline B0 & Nivel de la Curva de Rendimiento & Elaboración Propia \\
\hline B1 & Pendiente de la Curva de Rendimiento & Elaboración Propia \\
\hline EXPINFDIC & Expectativas de Inflación a Diciembre & Banco de la República de Colombia \\
\hline IMACO & Índice Mensual de Actividad Económica & Banco de la República de Colombia \\
\hline
\end{tabular}

Fuente: Elaboración Propia

\section{Estimación del vector autorregresivo}

Teniendo en cuenta que en la literatura sobre las modelaciones clásicos o puros, todas las variables dependen de todas, por lo tanto todas son modeladas endógenamente, además, atendiendo a que los estudiosos de las series de tiempo, interpretan que los proponentes de las metodologías sugieren que no es necesario diferenciar, restar tendencias o desestacionalizar las series, pues el objetivo primordial es descubrir las relaciones entre variables, más que estimaciones precisas de parámetros ${ }^{9}$; las series fueron modeladas en sus niveles naturales de obtención.

En armonía con lo anterior, se plantea un $\operatorname{VAR}(p)$ de la forma:

$$
X_{t}=C+\sum_{i=1}^{p} \theta_{i} X_{t-i}+\varepsilon_{t}
$$

\footnotetext{
${ }^{9}$ Ver por ejemplo Montenegro (2011) y Pérez (2006)
} 
Tabla 3.

Criterios de Elección de Rezagos

\begin{tabular}{cccccc}
\hline Rezago & $\mathbf{L o g}(\mathbf{L})$ & $\mathbf{P}(\mathbf{R V})$ & AIC & BIC & HQC \\
\hline $\mathbf{1}$ & 164,36626 & & $-2,673449$ & $\mathbf{- 2 , 1 7 6 7 5 8 *}$ & $-2,472059$ \\
\hline $\mathbf{2}$ & 190,53412 & 0,00001 & $-2,863595$ & $-1,969551$ & $\mathbf{- 2 , 5 0 1 0 9 3 *}$ \\
\hline $\mathbf{3}$ & 204,21914 & 0,03963 & $-2,818873$ & $-1,527476$ & $-2,295259$ \\
\hline $\mathbf{4}$ & 221,34788 & 0,00502 & $-2,839776$ & $-1,151026$ & $-2,155049$ \\
\hline $\mathbf{5}$ & 234,41482 & 0,05217 & 9,237174 & $-0,699358$ & $-1,939621$ \\
\hline $\mathbf{6}$ & 255,63692 & 0,00034 & $\mathbf{- 2 , 8 8 2 1 6 5 *}$ & $-0,39871$ & $-1,875214$ \\
\hline
\end{tabular}

Fuente: Elaboración propia

Tabla 4.

Vector Autorregresivo

\begin{tabular}{|c|c|c|c|c|}
\hline Variable & B0 & B1 & EXPINFDIC & IMACO \\
\hline \multirow{3}{*}{ B0(-1) } & 0.897182 & -0.273412 & 0.121515 & 0.000515 \\
\hline & $(0.08076)$ & $(0.10618)$ & $(0.03600)$ & $(0.00054)$ \\
\hline & [11.1090] & {$[-2.57505]$} & [3.37548] & [ 0.96130] \\
\hline \multirow{3}{*}{ B1(-1) } & -0.036208 & 0.761408 & 0.062338 & -0.000185 \\
\hline & $(0.05523)$ & $(0.07261)$ & $(0.02462)$ & $(0.00037)$ \\
\hline & {$[-0.65557]$} & 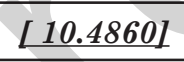 & 2.532111 & {$[-0.50636]$} \\
\hline \multirow{3}{*}{ EXPINFDIC(-1) } & 0.141125 & 0.300277 & 0.741852 & -0.001397 \\
\hline & $(0.13235)$ & $(0.17400)$ & $(0.05899)$ & $(0.00088)$ \\
\hline & [ 1.06630$]$ & [1.72572] & {$[12.5749]$} & {$[-1.59184]$} \\
\hline \multirow{3}{*}{ IMACO(-1) } & 4.754401 & 12.76913 & 2.620144 & 0.997424 \\
\hline & $(4.38456)$ & $(5.76440)$ & $(1.95441)$ & $(0.02907)$ \\
\hline & [ 1.08435$]$ & {$[2.21517]$} & {$[1.34063]$} & [34.3132] \\
\hline
\end{tabular}

Fuente: Elaboración propia

Donde $X_{t}^{\prime}=\left[\beta_{0 t}, \beta_{1 t}\right.$, EXPINFDIC $C_{t}, I M A$ $\left.C O_{t}\right]$ y se asume que el vector de errores esta independiente e idénticamente distribuido. El orden del VAR acorde al criterio Bayesiano de Schwarz ${ }^{10}$ podría ser estimado a un rezago, (Ver tabla 3).Con lo cual el vector se reescribirá así:

$X_{t}^{\prime}=\left[\beta_{0 t-1}, \beta_{1 t-1}, \beta_{2 t-1}, E X P I N F D I C_{t-1}, I M A C O_{t-1}\right]$

\footnotetext{
${ }^{10} \mathrm{El}$ hecho de que se atienda este criterio para modelar los rezagos del VAR obedece solo a la conveniencia de no sobre parametrizar el modelo.
}

Los resultados de la anterior modelación $V A R$ se muestran en la tabla 4 donde se evidencia que tanto las tasas de largo plazo como las expectativas de actividad económica, presentan un comportamiento exógeno al modelo, donde solo dependen significativamente de ellas mismas. Por su parte, la curvatura y las expectativas de inflación son bastante endógenas, en el sentido de que en términos estadísticos son significativamente explicadas por el modelo. 
La pendiente, que en términos de Alfaro (2009), puede ser interpretada como premio por plazo, y de alguna manera observa las tasas de mediano plazo, son determinadas estadísticamente por el contexto de la expectativas, y negativamente por las tasas de largo plazo. A su vez las expectativas de inflación a diciembre de cada año, son influenciadas significativamente tanto por las tasas de largo como de mediano plazo.

En el Anexo No 2, el grafico de las raíces inversas, da cuenta de la estabilidad del modelo, pues los puntos se encuentran dentro del círculo unitario.

\section{Identificación del VAR:}

\section{(funciones de impulso respuesta)}

Como quiera que la identificación en un modelo VAR se realiza mediante la ortogonalización de las perturbaciones aleatorias, transformando el modelo de manera que la matriz de varianzas covarianzas resultante sea diagonal (Perez, 2006), con esto el orden de las variables es de suma importancia para la identificación de los shocks, teniendo que ordenar las series de mayor a menor exogeneidad relativa. Pero la identificación de los shocks, mediante los impulsos generalizados técnicamente no exige la ortogonalización de los shocks ni ordenar las series respecto a su exogeneidad.

Se evidencia como las perturbaciones del nivel de la curva, afectan en forma ascendente, permanente y casi que de inmediato tanto a las desviaciones de las expectativas de inflación como a las de la actividad económica. A su vez los shocks de la pendiente se presentan después de tres períodos y en forma negativa sin mayor impacto sobre las desviaciones de amabas expectativas. Ver figura 11.
Respuesta de EXPINFDIC a B0
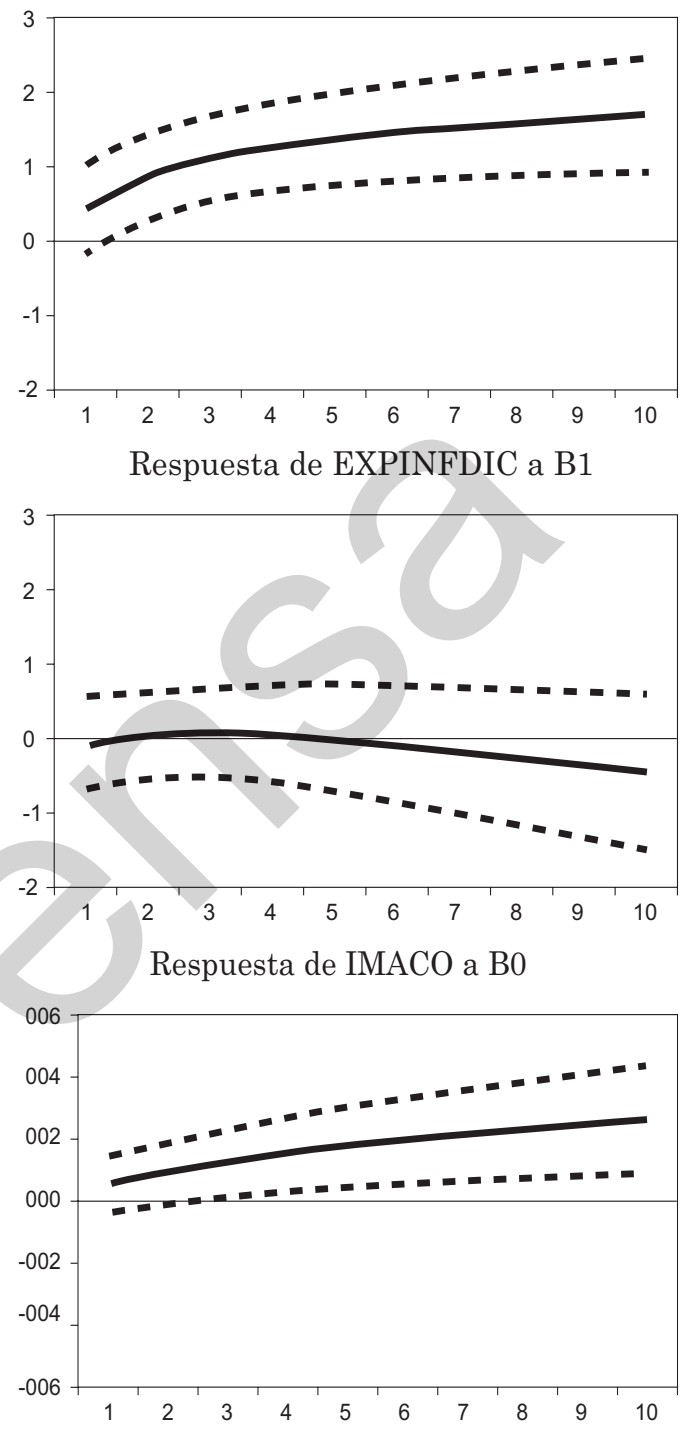

Respuesta de IMACO a B1

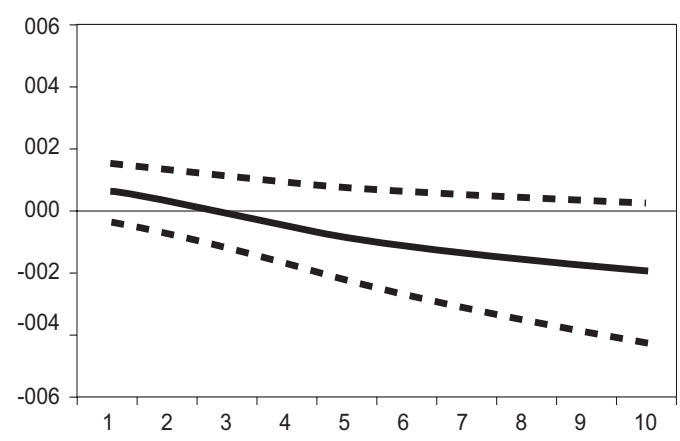

Figura 11. Respuesta de las Expectativas ante Desviaciones de las Tasas de Interés

Fuente: Elaboración propia 


\section{CONCLUSIONES}

En este trabajo, se estimó la estructura de tasas explotando las propiedades de las series de tiempo, en un contexto discreto (mensualmente) a partir de los Títulos de Tesorería de clase $\mathrm{B}$, evidenciando que en Colombia existe en el mercado de deuda pública la suficiente liquidez para ajustar dichas curvas, bajo este escenario discreto.

En el escenario de los típicos modelos afines o de factores, los resultados del ajuste de la estructura de tasas son similares a los obtenidos por Diebold \& Li (2006). Al acotar el parámetro que impone la no linealidad [op en este trabajo y $\lambda$ para Diebold \& Li (2006)] a un valor fijo para calibrar el modelo en forma lineal por mínimos cuadrados ordinarios, los resultados son muy similares. Así mismo, la estimación de vencimientos para tres periodos de la muestra, permite observar que el modelo se ajusta razonablemente bien a la dinámica real de las tasas.

El escenario del vector autorregresivo, permite inferir que las expectativas de inflación son más explicadas por los factores de las curvas de rendimiento, en donde las tasas de largo plazo tienen más peso que las de corto. Respecto al impacto de las perturbaciones de los factores de las curva de rendimiento, se evidencia que tienen efectos inmediatos, ascendentes y sostenidos sobre las desviaciones de las expectativas de inflación. Mientras que los shocks de la pendiente se manifiestan tres periodos después, en forma negativa y sin mayor impacto sobre las desviaciones de ambas expectativas.

\section{REFERENCIAS}

Alfaro, R. (2009). La Curva de Rendimiento bajo Nelson Siegel. Recuperado de: http://www.bcentral.cl/estudios/documentos-trabajo/pdf/dtbc531.pdf
Alfaro, R. (2011). Affine Nelson-Siegel Model.Economics Letters, 110(1), 1-3. doi: 10.1016/j.econlet.2010.10.003

Alfaro, R., Becerra, S. \& Sagner, A. (2011). Estimacion de la estructura de tasas nominales de Chile: aplicacion del modelo dinamico Nelson-Siegel. Econmía Chilena, 14(3), 57-74.

Cámaro, A., Henao, A. \& Mendez, E. (2005). Movimientos de la Curva de Rendimientos de TES Tasa Fija en Colombia. Innovar, 15(26), 122-133.

Christensen, J. H. E., Diebold, F. X., \& Rudebusch, G. D. (2010). The Affine Arbitrage-Free Class of Nelson-Siegel Term Structure Models.Recuperado de: http://www.frbsf.org/economic-research/files/wp07-20bk.pdf

Cox, J. C., Ingersoll, J. E. \& Ross, S. A. (1985). A theory of the term structure of interest rates. Econometrica, 53(2), 385-408.

Diebold, F. X. \& Li, C. (2006). Forecasting the Term Tructure of Government Bond Yields.Journal of Econometrics, 130(2), 337-364. doi: 10.1016/j. jeconom.2005.03.005

Diebold, F. X., Rudebusch, G. D., \& Aruoba, S. B. (2006). The Macroeconomy and the Yield Curve: a Dynamic Latent Factor Approach.Journal of Econometrics, 131(1-2), 309-338. doi: 10.1016/j.jeconom.2005.01.011

Dumrauf, G. L. (2015). Análisis cuantitativo de bonos. Argentina: Alfaomega. Recuperadp de: http://libroweb. alfaomega.com.mx/catalogo/analisis_ cuantitativo_bonos/libreacceso

Marin, J. \& Rubio, G. (2011). La Estructura Temporal de los Tipos de Interes en un Contexto de Ausencia de arbitraje. Recuperado de: https://books. google.com.co/books?id=tmZXGX40 $\mathrm{fZsC} \& \mathrm{pg}=\mathrm{PA} 61 \& \operatorname{lpg}=\mathrm{PA} 61 \& \mathrm{dq}=\mathrm{La}$ 
+Estructura+Temporal+de+los+Tip os +de+Interes+en+un+Contexto+d e+Ausencia + de + arbitraje\& source $=$ bl\&ots $=$ pdePkjDef $7 \&$ sig $=6 \mathrm{Y} 6 \mathrm{HaLo}$ LR_8exquQEtV3CSH3k2g\&hl=es$419 \& \mathrm{sa}=X \&$ ei $=a p K V V Z z n L M a X$ NpXxrvAF\&ved $=0 \mathrm{CBw}$ Q6AEwA $\mathrm{A} \# \mathrm{v}=$ onepage \& $\mathrm{q}=\mathrm{La} \% 20$ Estructura\%20Temporal\%20 de $\% 201$ os $\% 20$ Tipos\%20de\%20Interes\%20en\%20 un $\% 20$ Contexto\%20de\%20Ausencia\%20de\%20arbitraje\&f=false

Mascareñas, J. (2013). La Estructura Temporal de los Tipos de Interés. Recuperado de: http://pendientedemigracion.ucm.es/info/jmas/mon/07.pdf

Melo, L.\& Castro, G. (2010).Relacion Entre Variables Macro y la Curva de Rendimientos. Borradores de Economia, 605, 2-28. Recuperado de: http://www.banrep.gov.co/sites/ default/files/publicaciones/pdfs/borra605.pdf

Montenegro, A. (2011). Modelos Estacionrios Multivariados. Bogota: Editorial Universidad Nacional.

Nelson, C. R. \& Siegel, A. F. (1987). Parsimonious Modeling of Yield Curves. The Journal of Business, 60(4), 473489.

Perez, C. (2006). Econometria de las Series Temporales. Barcelona, España: P. P. Hall, Ed
Reveiz, A. \& Leon, C. (2008). Indice representativo del mercado de deuda publica interna: INXTES. Borradores de Economia, 488, 1-26. Recuperado de: http://www.banrep.gov.co/docum/ftp/ borra488.pdf

Rojas, J. C. (2007). La curva de rendimientos como predictor de expectativas macroeconómicas. Bogota: Serie Documentos Borradores de Investigación Universidad del Rosario, (93), 1-14.

Romero, P. A. \& Robles, M. D. F. (2003). Estructura temporal de los tipos de interes: teoria y evidencia empirica, Revista Australiana de Economia, 27, $7-47$.

Rudebusch, G. D. \& Wu, T. (2004). A MacroFinance Model of the Term Structure, Monetary Policy and the Economy. Recuperado de: http://www.frbsf.org/economic-research/files/wp03-17bk.pdf

Santana, J. (2008). La Curva de Rendimientos: una Revision Metodologica y nuevas Aproximaciones de Estimacion. Cuadernos de Economia, 27(48), 73-113.

Svensson, L. O. (1994). Estimating and Interpreting Forward Interest Rates Sweden 1992-1994. doi: 10.3386/w4871

Vasicek, O. (1977). An equilibrium characterization of the term structure. Journal of Financial Economics, 5, 177-188. 


\section{ANEXOS}

Anexo No. 1:

Test de Raiz Unitaria

\begin{tabular}{lccccc}
\hline \multirow{2}{*}{ Series } & \multicolumn{2}{c}{ ADF } & \multicolumn{2}{c}{ zPhillips-Perron } & KPSS (1\%=0,739-5\%=0,463 - 10\%=0,347\%) \\
\cline { 2 - 6 } & t-statistic & p-value & t-statistic & p-value & t-statistic \\
\hline B0 & $-2,004833$ & 0,2845 & $-1,9441$ & 0.3112 & 0,873712 \\
\hline B1 & $-2,470911$ & 0,1253 & $-2,23038$ & 0,1968 & $\mathbf{0 , 2 1 5 2 1 5}$ \\
\hline B2 & $\mathbf{- 4 , 0 5 9 2 4 2}$ & $\mathbf{0 , 0 0 1 7}$ & $\mathbf{- 4 , 0 6 5 9 3 4}$ & $\mathbf{0 . 0 0 1 6}$ & $\mathbf{0 , 2 3 0 6 8 5}$ \\
\hline EXPINFDIC & $-1,463342$ & 0,5486 & $-1,612206$ & 0,4731 & 0,792083 \\
\hline IMACO & $-2,83494$ & 0,0568 & $-1,557657$ & 0,5009 & 0,367047 \\
\hline
\end{tabular}

Fuente: Elaboración Propia

raídes inversas del VAR en relación al círculo unidad

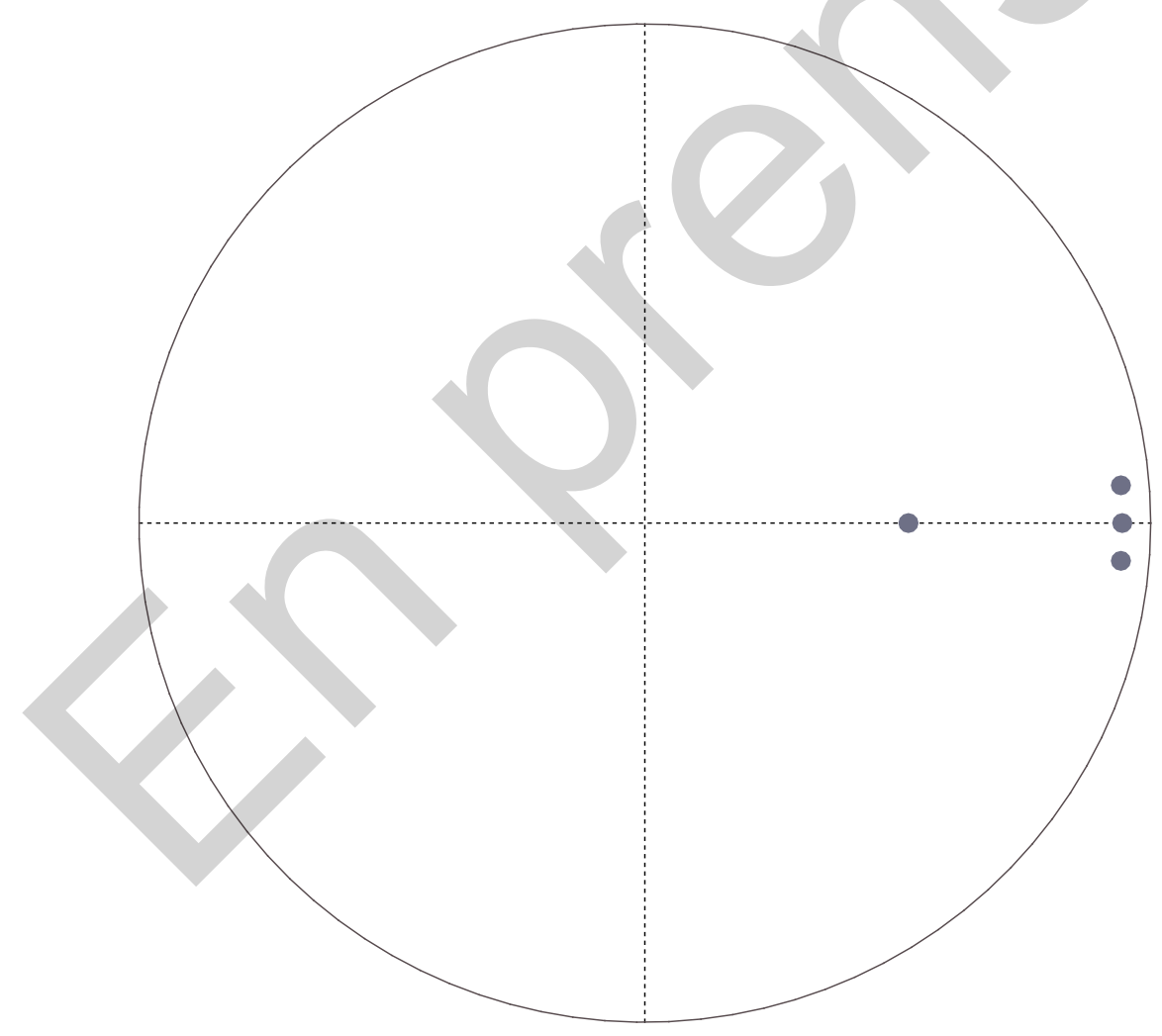

Anexo No. 2: Grafico de las Raíces Inversas del VAR

Fuente: Elaboración Propia 


$$
\left\langle v^{2}\right.
$$

